\title{
Distributed Maximum Lifetime Routing in Wireless Sensor Networks Based on Regularization
}

\author{
Vahid Shah-Mansouri and Vincent W.S. Wong \\ Department of Electrical \& Computer Engineering \\ The University of British Columbia, Vancouver, Canada \\ e-mail: \{vahids, vincentw\}@ece.ubc.ca
}

\begin{abstract}
The maximum lifetime routing problem in wireless sensor networks has received increasing attention in recent years. One way is to formulate it as a linear programming problem by maximizing the time at which the first node runs out of energy subject to the flow conservation constraints. The solutions in this problem correspond to the rates allocated to each link. In this paper, we first show that, under certain conditions, the solutions of this problem are not unique for some network topologies. Given the feasible solutions set, one can further define a secondary optimization problem by minimizing the end-to-end packet transfer delay or power consumption. Rather than solving two sequential optimization problems, in this paper, we propose the use of a regularization method which can jointly maximize the network lifetime and minimize another objective (e.g., packet delay). We describe the fully distributed implementation and provide performance comparisons with other algorithms.
\end{abstract}

\section{INTRODUCTION}

Recent advances in low power integrated circuits have sped up the development of various types of low cost wireless sensors, which are the building blocks of the wireless sensor networks (WSNs). In WSNs, each sensor node has the capability to sense the environment (e.g., temperature, pressure, light, acoustic) and process the data. In general, WSNs have an ad hoc topology and each node is capable of relaying the data towards the sink.

Most of the sensor nodes are battery powered. The limited amount of energy in each node is one of the bottlenecks. To overcome this problem, various energy aware or energyconstrained algorithms have been proposed in the literature (e.g., [1], [2], [3]). One simple approach is to minimize the power consumed to deliver a packet to the destination. The typical solution is to use the shortest path with link costs equal to the energy required in each link to transmit a packet [4]. Another approach is to maximize the lifetime of the network.

There are various ways to define the lifetime of a WSN. It can be defined as the time at which the first node runs out of its energy. Based on this definition, Chang and Tassiulas formulated the maximum lifetime routing problem as a linear programming problem [5]. A flow augmentation (FA) heuristic is used to solve the problem in a distributed manner. In [6], the work is extended by considering the case when the sequence of the generated data is unknown. In [7], Madan and Lall proposed both the partially distributed and fully distributed algorithms to solve the linear maximum lifetime problem in WSNs. In [8], the maximum lifetime problem is extended by considering a variable-length TDMA (Time Division Multiple Access) frame in the MAC (medium access control) layer. The problem is formulated as a convex optimization problem and a heuristic is used to solve it in a centralized manner. In [9], Brown et al. showed that maximizing the lifetime is equivalent to maximizing the traffic flow utility over the time. In [10], Hou et al. studied a two-tier WSN. They formulated the network lifetime problem for upper-tier aggregation and forwarding nodes as an optimization problem. In [11], Nama et al. studied the utility-lifetime tradeoff in maximum lifetime problem by considering the source rates as variables in the system. The objective is the utility maximization and a lifetimepenalty function is used to penalize large values of the inverse of the system lifetime. In [12], Dagher et al. proposed an iterative algorithm to find the Pareto optimal routing solution for the maximum lifetime problem.

For most of the maximum lifetime problems addressed in the literature (e.g., [5], [7]), the solutions correspond to the rates allocated to each link. In this paper, we first show that, under certain conditions, the solutions of this problem are not unique for some network topologies. Given the feasible solutions set, one can further define a secondary optimization problem by minimizing the end-to-end packet transfer delay or power consumption. Rather than solving two sequential optimization problems, in this paper, we propose a method to combine them as a single optimization problem. The regularization term does not change the complexity of the distributed method. The contributions of this paper are as follows:

- We propose the use of a regularization method which can jointly maximize the network lifetime and minimize another objective (e.g., packet delay, total power consumption).

- By using dual decomposition, the problem can be decomposed into several sub-problems (one for each node), which facilitates a fully distributed implementation.

- Results show that our proposed model not only can obtain the flow rates such that the network lifetime is maximized, it can also provide a lower average packet delay and power consumption when compared with other algorithms [5][7].

The rest of the paper is organized as follows: Section II describes the maximum lifetime problem and discusses the conditions under which the solutions are not unique. Section 
III introduces the regularization technique and the regularization functions. In Section IV, we present the maximum lifetime problem with regularization and describe a distributed algorithm based on dual decomposition. Results are presented in Section V. Conclusions are given in Section VI.

\section{BACKGROUND AND Motivations}

In this section, we first summarize the maximum lifetime routing problem formulation [5], [7]. We then provide examples to show that the optimal solutions are not unique. It is followed by a discussion on the second sequential optimization problem.

\section{A. Maximum Lifetime Routing Problem}

Consider a wireless sensor network. Let $V$ denote the set of sensor nodes, $N_{i}$ denote the set of neighbors of node $i \in V$, $S_{i}$ denote rate of data generated by node $i, x_{i j}$ denote the rate of data flows from node $i$ to node $j$ (i.e., aggregated rate). Let $S_{\text {sink }}$ denote the data to the sink. We have, $S_{\text {sink }}=$ $-\sum_{i \in V, i \neq \operatorname{sink}} S_{i}$. Let $E_{i}$ denote the initial energy of node $i$, $p_{i j}$ denote the average power consumed for transmission from node $i$ to $j$. Let $T_{i}(\mathbf{x})$ denote the lifetime of the node $i$ under data flow vector $\mathbf{x}=\left\{x_{i j}\right\}$. We have,

$$
T_{i}(\mathbf{x})=\frac{E_{i}}{\sum_{j \in N_{i}} p_{i j} x_{i j}}
$$

Sensor nodes are assumed to use TDMA for data transmission. Links which interfere with each other are not scheduled simultaneously. The maximum transmission rate and the maximum fraction of time allocated to each link are constant. The maximum flow for link $(i, j)$ is denoted by $R_{i j}$.

Given $S_{i}, E_{i}, p_{i j}$, and $R_{i j}$, the objective in the maximum lifetime routing problem is to maximize the time that the first node runs out of its energy, subject to the flow conservation constraints. We have,

$$
\begin{array}{ll}
\operatorname{maximize} & \min _{i \in V} T_{i}(\mathbf{x}) \\
\text { subject to } & \sum_{j \in N_{i}}\left(x_{i j}-x_{j i}\right)=S_{i}, \quad \forall i \in V \\
& 0 \leq x_{i j} \leq R_{i j}, \quad \forall i \in V, \forall j \in N_{i}
\end{array}
$$

Problem (2) can be converted to the following linear problem by a change of variable:

$$
\begin{array}{cll}
\text { minimize } & q & \\
\text { subject to } & \sum_{j \in N_{i}}\left(x_{i j}-x_{j i}\right)=S_{i}, \quad \forall i \in V \\
& \sum_{j \in N_{i}} p_{i j} x_{i j} \leq q E_{i}, \quad \forall i \in V \\
& 0 \leq x_{i j} \leq R_{i j}, \quad \forall i \in V, \forall j \in N_{i}
\end{array}
$$

where $q$ is the upper bound on the inverse of the lifetime of all the nodes in the network. The optimal value (minimal value) is denoted by $q^{\star}$ and is achieved at an optimal solution $\mathbf{x}^{\star}$.
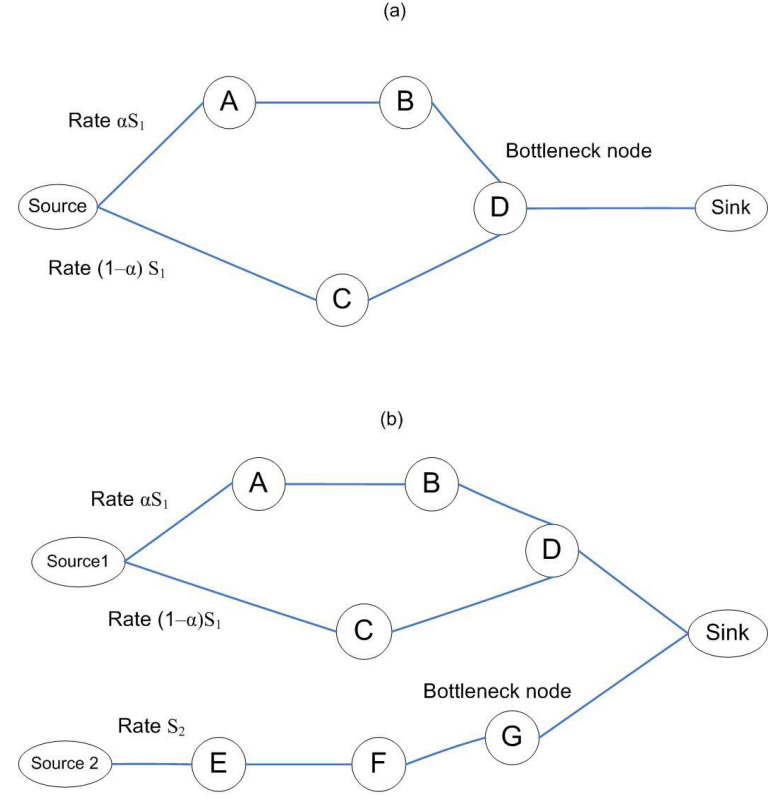

Fig. 1. (a) A WSN with one source and a sink; (b) A WSN with two sources and a sink.

\section{B. Multiple Optimal Solutions}

Problem (3) is a linear programming problem. The feasible region is the collection of all feasible solutions $\mathbf{x}$ which satisfy the constraints in (3). When the feasible region is not empty and the optimal value is bounded, then there will be either one optimal solution or multiple optimal solutions. When problem (3) has one optimal solution, it is a corner point solution. A corner point solution is an extreme point of the polyhedron constructed by the constraints. When problem (3) has multiple optimal solutions, they are in a hyperplane constructed by several constraints. Mathematically, an optimization problem which has a unique optimal solution is called a well-posed problem (and is called an ill-posed problem otherwise).

Consider the first example shown in Fig. 1 (a). Nodes $A, B$, $C$, and $D$ have equal initial energy. The source and sink nodes have higher initial energy. The source generates data at the rate of $S_{1}$. There are two paths between the source and sink nodes. The first path (called $P_{1}$ ) is via intermediate nodes $A, B$, and $D$. The second path (called $P_{2}$ ) is via intermediate nodes $C$ and $D$. Path $P_{1}$ carries $\alpha S_{1}$ proportion of source traffic. Path $P_{2}$ carries $(1-\alpha) S_{1}$ proportion of source traffic. We define a bottleneck node as the one which has the minimum lifetime in the network. Suppose node $D$ is the bottleneck node. Thus, the inequality constraint in problem (3) is active at node $D$ at the optimum point. That is,

$$
\sum_{j \in N_{D}} p_{D j} x_{D j}=q^{\star} E_{D}
$$

where $q^{\star}$ is the optimal value. Since all other constraints are satisfied as long as the source sends $\alpha S_{1}$ proportion of traffic via path $P_{1}$ and $(1-\alpha) S_{1}$ proportion of traffic via path $P_{2}$ for $0<\alpha<1$, the optimal solutions $\mathrm{x}^{\star}$ are not unique. 
Consider the second example shown in Fig. 1 (b). In this example, one node along the path between source 2 and the sink is the bottleneck. Similarly, source 1 can transmit a portion of its data $S_{1}$ via the two available multipaths and this portion can vary within a certain range. Thus, the optimal solutions $\mathbf{x}^{\star}$ are also not unique in this example.

\section{The Second Optimization Problem}

For the above examples, we show that for a WSN with multiple sources sending data to the sink, although the optimal value (i.e., maximum lifetime) can be determined by solving either problem (2) or (3), the optimal solutions (i.e., routing paths and the corresponding data rates) are not unique. This gives an opportunity to further optimize another objective function.

To this end, let $X^{\star}$ denote the set of optimal data flow vectors such that each vector in this set is the optimal solution in problem (3). Thus, a second optimization problem can be defined such that the variables are restricted to be the optimal solutions in problem (3). The second optimization problem can be defined as:

$$
\text { minimize } \phi(\mathbf{x}), \quad \text { subject to } \mathbf{x} \in X^{\star}
$$

where $\phi(\mathbf{x})$ can be either a cost function, a delay function, or a function used to model the power consumption. In addition, the term $-\phi(\mathbf{x})$ can be interpreted as a utility function. Since the constraints in problem (3) are either affine or convex functions, in order to ensure that problem (4) has a unique solution, a necessary and sufficient condition is that $\phi(\mathbf{x})$ be a strictly convex function.

In the next section, we provide the background to combine problems (3) and (4) as a single optimization problem.

\section{Exact Regularization of Linear Program}

Consider the following linear programming problem with variable $\mathrm{x}$ :

$$
\begin{array}{cl}
\operatorname{minimize} & f_{0}(\mathbf{x}) \\
\text { subject to } & f_{i}(\mathbf{x}) \geq 0, \quad i=1, \ldots, m \\
& \mathbf{x} \succeq \mathbf{0}
\end{array}
$$

where $f_{0}, f_{i}$ 's are linear functions. The optimal value (minimal value) is denoted by $p^{\star}$ and is achieved at an optimal solution $\mathbf{x}^{\star}$. That is, $p^{\star}=f_{0}\left(\mathbf{x}^{\star}\right)$.

The second optimization problem is defined as:

$$
\begin{array}{cl}
\operatorname{minimize} & \phi(\mathbf{x}) \\
\text { subject to } & f_{i}(\mathbf{x}) \geq 0, \quad i=1, \ldots, m \\
& f_{0}(\mathbf{x}) \leq p^{\star} \\
& \mathbf{x} \succeq \mathbf{0}
\end{array}
$$

where the domain of $\mathrm{x}$ is the set of optimal solutions in (5), and $\phi(\mathbf{x}): \mathcal{R}^{n} \rightarrow \mathcal{R}$ can either be a linear or nonlinear function. The level set of $\phi(\mathbf{x})$ is nonempty and bounded.

The nonlinear regularization (or perturbation) of linear programs was first proposed by Mangasarian et al. [13]. They showed that solving (5) and (6) sequentially is equivalent to solving the following optimization problem:

$$
\begin{array}{cl}
\operatorname{minimize} & f_{0}(\mathbf{x})+\delta \phi(\mathbf{x}) \\
\text { subject to } & f_{i}(\mathbf{x}) \geq 0, \quad i=1, \cdots, m \\
& \mathbf{x} \succeq \mathbf{0}
\end{array}
$$

Mangasarian et al. [13] proved that for all values of $\delta$ below some positive threshold, the optimal solutions of the regularized problem (7) are also the optimal solutions in problems (5) and (6). Recently in [14], Friedlander et al. showed that this threshold is the inverse of the Lagrange multiplier of the second inequality constraint in problem (6). They proved that problem (6) always has a Karush-Kuhn-Tucker (KKT) point. Thus, this threshold always exists.

\section{A. Regularization Function}

For applications in WSNs, the regularization function $\phi(\mathbf{x})$ is aimed to satisfy the following properties:

1) It is a strictly convex function.

2) It is a separable function.

3) It has a physical interpretation (e.g., end-to-end delay of a path).

Property (1) ensures that the problem (7) is a well-posed problem. Property (2) allows problem (7) to be solved in a distributed manner. Separability implies that $\phi(\mathbf{x})$ is the sum of some functions $\phi_{i}$ and each local function $\phi_{i}$ only depends on the information around node $i$. Finally, since $\phi(\mathbf{x})$ is used as part of the objective function, property (3) ensures that it has a physical meaning.

A common form of regularization is proposed by Tikhonov [15][16] where $\phi(\mathbf{x})=\|\mathbf{x}\|_{2}^{2}$. This function was also used in [7]. Although this function satisfies properties (1) and (2), it does not provide a physical meaning.

In the following, we propose two regularization functions which satisfy the above three properties.

1) Delay Function: The end-to-end packet delay between a source and a sink depends on the number of hops of the path and the queuing delay in each intermediate node along the path. For most of the WSN's applications, since the data rate is low and data are also aggregated along the path, it is reasonable to assume that the queuing delay is not a dominant factor and the number of hops is more important.

Assume that each sensor node $i$ knows the geographical locations of itself, its neighbors $j \in N_{i}$, and the sink via some localization algorithms [17]. Thus, it can determine the distance $d_{j, \text { sink }}$ between node $j$ and the sink. We define:

$$
h_{i j}=\frac{d_{j, \operatorname{sink}}}{d_{i, \operatorname{sink}}}, \quad i \in V, j \in N_{i}
$$

For a given node $i$, if its neighbor $j \in N_{i}$ is farther from the sink than another neighbor $k \in N_{i}$, then $h_{i j}>h_{i k}$. We propose the local delay function at node $i$ as:

$$
\phi_{i}(\mathbf{x})=\sum_{j \in N_{i}} h_{i j}^{2} x_{i j}^{2}, \quad i \in V
$$


Node $i$ is able to forward its data towards the sink through some its neighbors. It sends data through the neighbors with lower $h_{i j}$ (or equivalently through a subset of neighboring nodes that are closer to the sink). Taking the square of each term ensures that the function $\phi_{i}$ is strictly convex. This leads to a suboptimality. We show the amount of suboptimality in performance comparison section.

The regularization function for delay is:

$$
\phi(\mathbf{x})=\sum_{i \in V} \phi_{i}(\mathbf{x})=\sum_{i \in V} \sum_{j \in N_{i}} h_{i j}^{2} x_{i j}^{2}
$$

The above function ensures that most of the data traffic is routed through the minimum hop path on average.

2) Minimizing the Total Power Consumption: A common objective function to minimize the total power consumption in the WSN is $\sum_{i \in V} \sum_{j \in N_{i}} p_{i j} x_{i j}$. Since the above function is not strictly convex, we take the square in each term and define the regularization function for power as:

$$
\phi(\mathbf{x})=\sum_{i \in V} \phi_{i}(\mathbf{x})=\sum_{i \in V} \sum_{j \in N_{i}} p_{i j}^{2} x_{i j}^{2}
$$

The function in (11) is strictly convex and separable. Each node $i$ chooses to forward data through its neighbors $j$ which require less transmission power $p_{i j}$.

\section{Distributed Algorithm of Regularized PROBLEM}

In this section, we first present the primal problem which is decomposable. For distributed implementation, we then use dual decomposition to separate the primal problem into several sub-problems, one for each node.

Based on the results from Section III, problems (3) and (4) can be combined as a single optimization problem. To ensure that the primal problem is decomposable, we use the similar technique as in [7]. For the objective function in (3), $q$ is replaced by $\sum_{i \in N_{i}} q_{i}^{2}$ which is decomposable. An additional constraint $q_{i}=q_{j}$ (for all $i \in V, j \in N_{i}$ ) is included to make sure that all $q_{i}$ 's are equal.

The new primal problem which combines problems (3) and (4) is as follows:

$$
\begin{array}{lll}
\operatorname{minimize} & \sum_{i \in V}\left(q_{i}^{2}+\delta \phi_{i}(\mathbf{x})\right) & \\
\text { subject to } & \sum_{j \in N_{i}}\left(x_{i j}-x_{j i}\right)=S_{i}, \quad \forall i \in V \\
& \sum_{j \in N_{i}} p_{i j} x_{i j} \leq q_{i} E_{i}, \quad \forall i \in V \\
& q_{i}=q_{j}, \quad \forall i \in V, \forall j \in N_{i} \\
& 0 \leq x_{i j} \leq R_{i j}, \quad \forall i \in V, \forall j \in N_{i}
\end{array}
$$

where $\sum_{i \in V} \phi_{i}(\mathbf{x})$ is the regularization term for either delay objective (10) or power objective (11). Problem (12) is a convex optimization problem and it can be solved by using the dual decomposition approach. We first introduce the Lagrange multipliers $(\nu$ and $\gamma)$ for the equality constraints in (12). The other constraints are local constraints in each node and need not be relaxed. The Lagrangian is:

$$
\begin{aligned}
L(\mathbf{q}, \mathbf{x}, \nu, \gamma)= & \sum_{i \in V}\left(q_{i}^{2}+\delta \phi_{i}(\mathbf{x})\right) \\
& +\sum_{i \in V} \nu_{i}\left(\sum_{j \in N_{i}}\left(x_{i j}-x_{j i}\right)-S_{i}\right) \\
& +\sum_{i \in V} \sum_{j \in N_{i}} \gamma_{i j}\left(q_{i}-q_{j}\right) \\
= & -\sum_{i \in V} \nu_{i} S_{i}+\sum_{i \in V}\left(q_{i}^{2}+\delta \phi_{i}(\mathbf{x})\right. \\
& \left.+q_{i} \sum_{j \in N_{i}}\left(\gamma_{i j}-\gamma_{j i}\right)+\sum_{j \in N_{i}} x_{i j}\left(\nu_{i}-\nu_{j}\right)\right) .
\end{aligned}
$$

From the Lagrangian, the dual function and the dual problem can be defined. A subgradient algorithm can be used to solve the dual problem. We use the following distributive algorithm to solve problem (12).

\section{Distributed algorithm for each source $i \in V$ :}

Given $\nu(t), \gamma(t)$, and the local information $\left(p_{i j}, E_{i}, R_{i j}\right)$, each node $i$ updates the variables $q_{i}(t)$ and $x_{i j}(t)$ for $j \in N_{i}$ by solving the following problem at each iteration $t$ :

$$
\begin{aligned}
\operatorname{minimize} & q_{i}^{2}(t)+\delta \phi_{i}(\mathbf{x}(t))+q_{i}(t) \sum_{j \in N_{i}}\left(\gamma_{i j}(t)-\gamma_{j i}(t)\right) \\
& +\sum_{j \in N_{i}} x_{i j}(t)\left(\nu_{i}(t)-\nu_{j}(t)\right) \\
\text { subject to } & \sum_{j \in N_{i}} p_{i j} x_{i j}(t) \leq q_{i}(t) E_{i} \\
& 0 \leq x_{i j}(t) \leq R_{i j}, \quad \forall j \in N_{i}
\end{aligned}
$$

The Lagrange multipliers $\nu_{i}(t)$ and $\gamma_{i j}(t)$ at each iteration $t$ are updated based on following equations:

$$
\nu_{i}(t+1)=\nu_{i}(t)-\alpha(t)\left(S_{i}-\sum_{j \in N_{i}}\left(x_{i j}(t)-x_{j i}(t)\right)\right),
$$

and

$$
\gamma_{i j}(t+1)=\gamma_{i j}(t)-\alpha(t)\left(q_{j}(t)-q_{i}(t)\right), \forall j \in N_{i}
$$

where $\alpha(t)$ is a positive step size and is chosen as $\frac{1}{t+1}$. Furthermore, $x_{i j}(t)$ and $q_{i}(t)$ are solutions of problem (13).

In summary, at each iteration, node $i$ updates the values of $q_{i}$ and $x_{i j}$ based on (13). The values of the Lagrange multipliers are updated based on (14) and (15). Node $i$ then exchanges the updated values of $q_{i}, x_{i j}, \nu_{i}$, and $\gamma_{i j}$, with its neighbors $j \in N_{i}$.

\section{Performance Comparisons}

In this section, we compare our distributed regularized algorithm with the flow augmentation heuristic [6] and the fully distributed algorithm proposed in [7].

In the first experiment, 36 nodes are randomly deployed in a square area with each side equal to 70 unit. The maximum 


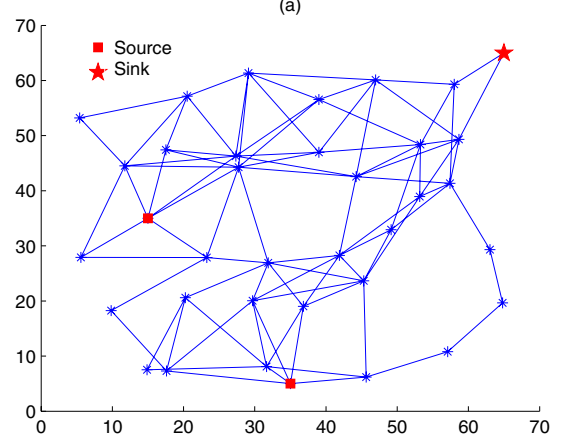

(b)

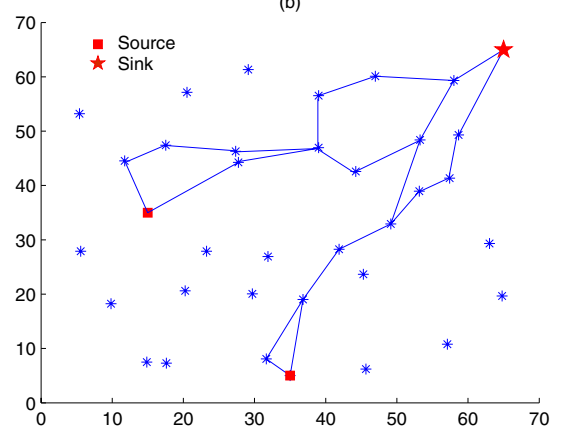

(c)

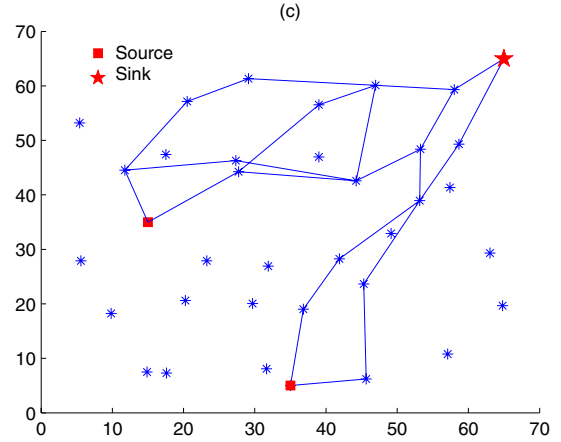

Fig. 2. Routing paths for different problems: (a) Fully distributed method [7]; (b) Regularized problem with power term; (c) Regularized problem with delay term.

transmission radius of each node is 15 unit. Two nodes are randomly selected as sources. The sink node is located at the top right corner in the network. We assume a deterministic path loss model. The power consumed for transmission of one bit from node $i$ to node $j$ (i.e., $p_{i j}$ ) is $\beta_{1}+\beta_{2} d^{4}$, where $d$ is the physical distance. We choose $\beta_{1}=1$ and $\beta_{2}=0.1$. For the regularization method, we select $\delta=10^{-5}$. Each source generates 100 bytes/sec of information. The initial energy of each source node $E_{S}$ is $30 \mathrm{~J}$ while the initial energy of each intermediate node $i$ (i.e., $E_{i}$ ) is $10 \mathrm{~J}$.

In the flow augmentation heuristic, the cost of each link is determined by [6]:

$$
\text { cost }_{i j}=p_{i j} \varepsilon_{i}^{-30} E_{i}^{-30}
$$

where $\varepsilon_{i}$ is the residual energy after each packet transmission. The packet generation rate is 1 packet/sec and each packet is 100 bytes.

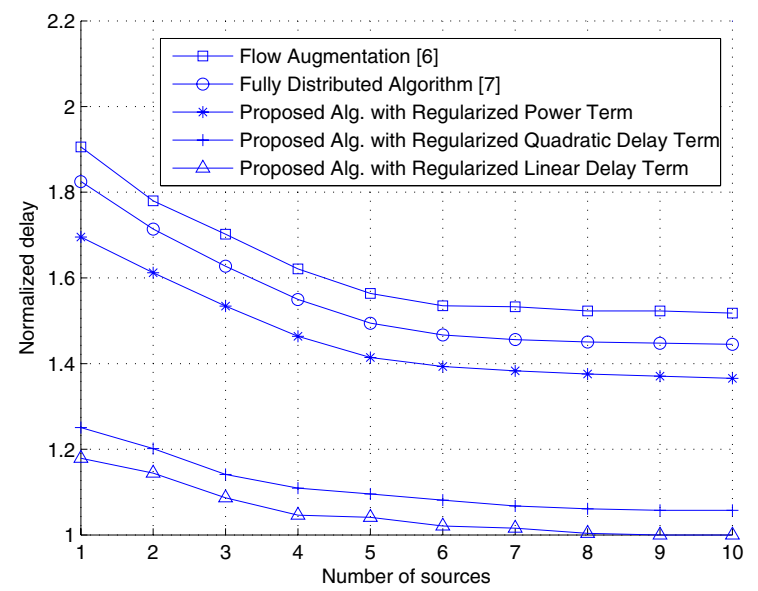

Fig. 3. Normalized average end-to-end delay by using different algorithms.

Fig. 2 depicts the routing paths by using different maximum lifetime routing algorithms. Fig. 2 (a) shows the routing based on the fully distributed method [7], Fig. 2 (b) shows the routing of the regularized program with power regularization term, and Fig. 2 (c) shows the routing with delay regularization term. Although all three algorithms provide the same maximum lifetime of the network, results in Fig. 2 (a) show that almost all the nodes are involved in routing while the results in Figs. 2 (b) and (c) show that only a subset of nodes are involved in routing between the two sources and the sink. Note that we do not include the results for the flow augmentation heuristic [6] as the results are similar to that in Fig. 2 (a) (i.e., the routing paths are the same while the data rate of the links are different).

In the next experiment, we present the results for networks with a larger number of nodes. 200 nodes are randomly deployed in a square region with each side equal to 100 unit. The maximum transmission radius of each node is 5 unit. There is one sink in the network. The number of sources varies in each simulation run. Other values of the parameters in the system are the same as in the previous experiment. The topology is changed randomly after each simulation run and the results are averaged over 1000 simulation runs.

Fig. 3 shows the results for the normalized average delay. The delay is defined as the average number of hops between each source and the sink. The average values are normalized by the value obtained from the regularized problem with linear delay term. Quadratic delay term is the term that we used to obtain a strictly convex regularization term. The quadratic term is obtained by taking square from each term in linear regularization term.

Results show that our proposed algorithm with regularized delay term provides a lower normalized delay than the flow augmentation heuristic [6] and the fully distributed algorithm [7]. This figure also shows that the suboptimality produced by using strictly convex term is less than $10 \%$.

Fig. 4 shows the results for the normalized average power. 


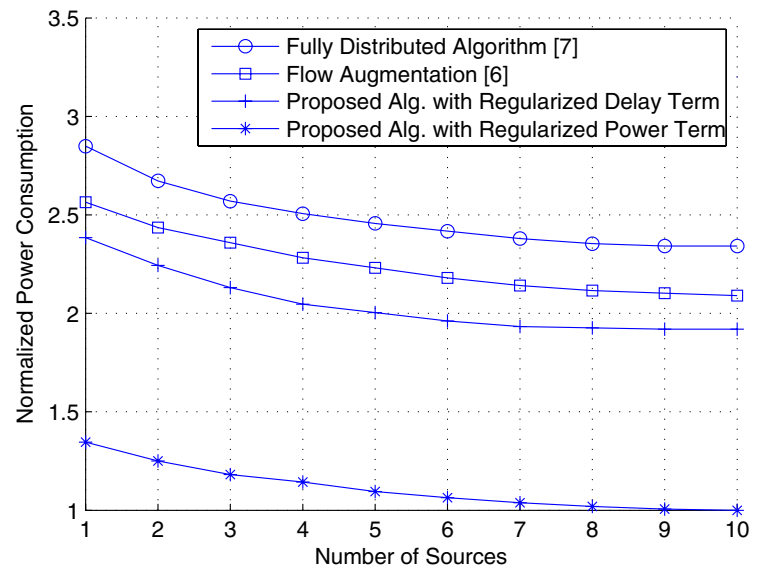

Fig. 4. Normalized average total power consumption by using different algorithms.

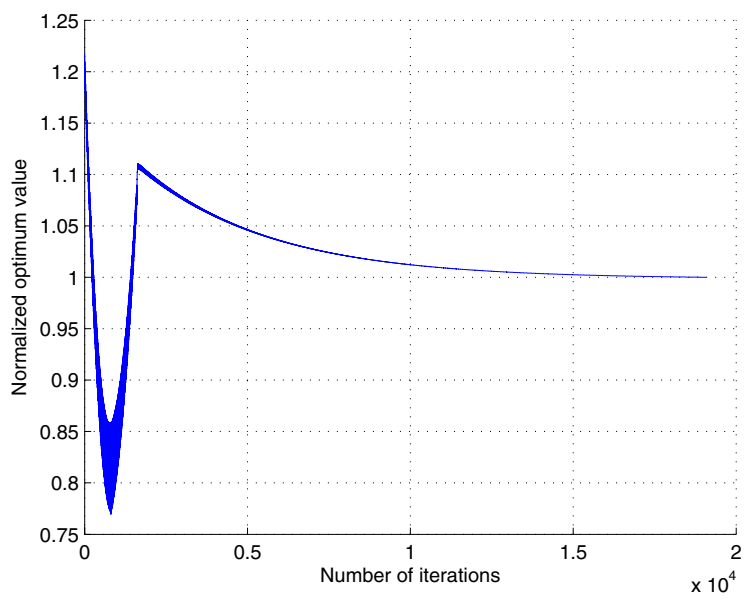

Fig. 5. Convergence of the optimal value in the network with one source and 200 nodes.

The total power is defined as the average total power consumed along each path from the source node to the sink. The average values are normalized by the value obtained from the regularized problem with power term. Results show that our proposed algorithm with regularized power term provides a lower normalized power consumption than the other two schemes.

Fig. 5 shows the lifetime of one of the topologies versus the iteration number used in our distributed implementation of our proposed regularized problem. The distributed algorithm converges within $5 \%$ of the optimal point after 5,000 iterations, and it converges to the optimal point after 15,000 iterations.

\section{CONCLUSIONS}

In this paper, we first showed that the maximum lifetime routing problem in general case may lead to multiple optimal solutions. Different solutions may lead to different network behaviors such as end-to-end delay or total power consumption. To choose a unique solution, a second optimization problem may be necessary. We proposed the use of regularization to combine two sequential optimization problems as a single problem. We also proposed two regularization functions for delay and total power consumption. We described the distributed algorithm for the regularized problem via dual decomposition. When compared our proposed algorithm with those in [6] and [7], although all three provide the same optimal lifetime value, our proposed algorithm can further reduce either the average end-to-end delay or the total power consumption.

\section{ACKNOWLEDGEMENT}

This work is supported by the Natural Sciences and Engineering Research Council of Canada (NSERC). The authors would like to thank Michael Friedlander for discussions on this paper.

\section{REFERENCES}

[1] V. Rodoplu and T. Meng, "Minimum energy mobile wireless networks," IEEE J. on Selected Areas in Communications, vol. 17, pp. 1333-1344, Aug. 1999.

[2] R. C. Shah and J. M. Rabaey, "Energy aware routing for low energy ad hoc sensor networks," in Proc. of IEEE WCNC, Orlando, FL, Mar. 2002.

[3] A. Goldsmith and S. Wicker, "Design challenges for energy-constrained ad hoc wireless networks," IEEE Trans. on Wireless Communications, vol. 9, pp. 8-27, Aug. 2002.

[4] S. Singh, M. Woo, and C. Raghavendra, "Power-aware routing in mobile ad hoc networks," in Proc. of ACM MobiCom, Dallas, Texas, Oct. 1998

[5] J. Chang and L. Tassiulas, "Routing for maximum system lifetime in wireless ad-hoc networks," in Proc. of Allerton Conference Communication, Control, and Computing, Urbana, Illinois, Sept. 1999.

[6] —- "Maximum lifetime routing in wireless sensor networks," IEEE/ACM Trans. on Networking, vol. 12, pp. 609-619, Aug. 2004.

[7] R. Madan and S. Lall, "Distributed algorithms for maximum lifetime routing in wireless sensor networks," IEEE Trans. on Wireless Communications, vol. 5, pp. 2185-2193, Aug. 2006.

[8] R. Madan, S. Cui, S. Lall, and A. Goldsmith, "Cross-layer design for lifetime maximization in interference-limited wireless sensor networks," IEEE Trans. on Wireless Communications, vol. 5, pp. 3142-3152, Nov. 2006.

[9] T. Brown, H. Gabow, and Q. Zhang, "Maximum flow-life curve for a wireless ad hoc network," in Proc. of ACM MobiCom, Long Beach, CA, Oct. 2001.

[10] Y. T. Hou, Y. Shi, J. Pan, and S. F. Midkiff, "Maximizing the lifetime of wireless sensor networks through optimal single-session flow routing," IEEE Trans. on Mobile Computing, vol. 5, pp. 1255-1266, Sept. 2006.

[11] H. Nama, M. Chiang, and N. Mandayam, "Optimal utility-lifetime trade-off in wireless sensor network: Characterization and distribution algorithms," submitted to IEEE Trans. on Wireless Communications, 2007.

[12] J. C. Dagher, M. W. Marcellin, and M. A. Neifeld, "A theory for maximizing the lifetime of sensor networks," IEEE Trans. on Communications, vol. 55, pp. 323-332, Feb. 2007.

[13] O. Mangasarian and R. Meyer, "Nonlinear perturbation of linear programs," SIAM Journal on Control and Optimization, vol. 17, pp. 745752, 1977.

[14] M. Friedlander and P. Tseng, "Exact regularization of convex programs," Tech. Rept. TR-2006-26., 2006, Available online: http://www.cs.ubc.ca/ mpf/assets/files/papers/cpreg.pdf.

[15] A. Tikhonov and V. Arsenin, Solutions of Ill-posed Problems. V. H. Wilston and Sons, Washington, DC, Translated from Russian, 1977.

[16] S. Boyd and L. Vandenberghe, Convex Optimization. Cambridge University Press, 2004.

[17] V. Vivekanandan and V. W. S. Wong, "Concentric anchor-beacons (CAB) localization for wireless sensor networks," in Proc. of IEEE International Conference on Communications (ICC), Istanbul, Turkey, June 2006. 